Research Paper

\title{
Safety and outcomes of volumetric modulated arc therapy in the treatment of patients with inoperable lung cancer
}

\author{
Xiance Jin ${ }^{1 *}$, Baochai Lin ${ }^{1 *}$, Didi Chen ${ }^{1 *}$, Lili Li ${ }^{1}$, Ce Han ${ }^{1}$, Yongqiang Zhou ${ }^{1}$, Xiaomin Zheng1, Changfei \\ Gong1, Mengfeng Chen², Congying Xie ${ }^{1 凶}$ \\ 1. Radiation and Medical Oncology, the first Affiliated Hospital of Wenzhou Medical University, Wenzhou, China,325000, \\ 2. Respiratory Department, Third People's Hospital of Yueqing, Wenzhou, China, 325600 \\ *authors contribute equally \\ $\square$ Corresponding author: Congying Xie, Ph.D., Radiotherapy and Chemotherapy Department, the 1st Affiliated Hospital of Wenzhou Medical University, \\ Wenzhou, China, 325000. Phone: 0086-577-88069316; Fax: 0086-577-55578999-611881; Email: wzxiecongying@163.com \\ (c) Ivyspring International Publisher. This is an open access article distributed under the terms of the Creative Commons Attribution (CC BY-NC) license \\ (https://creativecommons.org/licenses/by-nc/4.0/). See http://ivyspring.com/terms for full terms and conditions.
}

Received: 2018.11.05; Accepted: 2019.04.14; Published: 2019.06.02

\begin{abstract}
Background: Published data on the effects and toxicities of volumetric modulated arc therapy (VMAT) in the management of inoperable lung cancer are scarce.

Materials and methods: The clinical outcomes and pulmonary toxicities of 134 patients with consecutive inoperable lung cancer who underwent VMAT from March 2011 to September 2016 were retrospectively reviewed. The dosimetric and characteristic factors associated with acute radiation pneumonitis (RP) and pulmonary fibrosis were evaluated with univariate and multivariate analysis.

Results: The average prescription doses to these 134 patients were $57.07 \pm 6.27$ Gy (range 52-64 Gy). The overall median follow-up time was 18.6 months (range, 2-45 mo), with a median follow-up time for the surviving patients of 20 months (range, 7-45 mo). The 2-year progression-free survival (PFS) and overall survival (OS) for all patients were $18.2 \%$ and $38.4 \%$, with a median PFS and OS of 7.6 months and 18.6 months, respectively. The percent of patients with grade III/higher RP and pulmonary fibrosis were $10.5 \%$ and $9.0 \%$, respectively. $\mathrm{V} 13(p=0.02)$ and age $(p=0.02)$ were independently associated with acute $\mathrm{RP}$ according to multivariate analysis. The constraints for lung dosimetric metrics $\mathrm{V} 10, \mathrm{~V} 13, \mathrm{~V} 20$ and $\mathrm{V} 30$ were approximately $49 \%, 41 \%, 26 \%$ and $17 \%$ in VMAT treatment of lung cancer to limit the RP rate $<10 \%$.

Conclusion: VMAT can be delivered safely with acceptable acute and late toxicities for lung cancer patients. Lung dosimetric metrics were valuable in predicting acute RP. A lung V1 3 constraint of $40 \%$ was helpful to limit the RP rate $<10 \%$ in VMAT treatment of lung cancer patients.
\end{abstract}

Key words: Lung cancer; Volumetric modulated arc therapy; Radiation pneumonitis; Disease free survival; Overall survival

\section{Introduction}

Radiotherapy is an important modality in the treatment of lung cancer. More than $50 \%$ of lung cancer patients received radiotherapy at some point during their treatment. ${ }^{1}$ For radical purposes, the ultimate goal of radiotherapy is to achieve local tumor control while sparing the surrounding normal tissue to limit toxicities. Currently, intensity-modulated radiotherapy (IMRT) is applied routinely in the management of both early stage and locally advanced non-small-cell lung cancer (NSCLC) in radiation centers across the world due to its ability to sculpt high-dose volume around the site of disease and thereby spare adjacent organs at risk (OAR).2,3 IMRT allows the treatment of target volumes previously considered too large for a radical dose and permits safer dose escalation to the tumor. ${ }^{4}$ A review article demonstrated that IMRT can be delivered safely with acceptable acute and late toxicities compared with 
three-dimensional (3D) conformal radiotherapy (CRT). ${ }^{5}$ Crucially retrospective studies also reported an advantage on disease-free survival (DFS) and overall survival (OS) over those achieved with conventional 3D-CRT.6,7

Despite the advantages of IMRT, concerns on the effects of intra-fraction motion had been raised, because IMRT requires multiple fixed angle beams that can increase the treatment delivery time. ${ }^{8}$ Although image-guided radiation therapy has improved the patient positioning accuracy, it often requires more time in treatment and increased radiation to the patient due to imaging before each treatment session. ${ }^{9}$ Furthermore, a higher number of monitor units (MUs) in IMRT plans could potentially increase the low dose radiation exposure of patients, and this has led to concerns of increased risk of secondary radiation-induced malignancies, especially for pediatric patients. ${ }^{10,11}$

Volumetric modulated arc therapy (VMAT) is an new form of IMRT with continuous changeable dose rate, gantry speed, and dynamic multileaf collimator movement. ${ }^{12}$ The major advantages of VMAT over IMRT are the decreased MU and treatment delivery time.13,14 A shorter treatment delivery time per fraction is particularly critical for tumors with a moving target such as lung cancer as the intra-fraction motion was found to increase with time. ${ }^{15}$ The published data on VMAT (RapidArc and SmartArc) planning studies for lung cancer showed that VMAT techniques have clear superiority over 3DCRT with regard to improving dose conformity and sparing of OARs. ${ }^{16}$

On the other hand, published clinical data on safety and clinical outcomes of VMAT in lung cancer treatment are scarce. Therefore, the purpose of this study is to investigate the effects, and the acute and late pulmonary toxicities of VMAT (sequential/ concurrent chemotherapy) in the treatment of patients with inoperable lung cancer.

\section{Methods and Materials}

\section{Patient characteritics}

Between March 2011 and September 2016, a total of 134 consecutive patients with inoperable lung cancer were treated by VMAT with or without concurrent chemotherapy in our hospital. Concurrent chemotherapy was administered with a combination of cisplatin and etoposide or carboplatin and paclitaxel. The retrospective review was approved by the Institutional Review Board (IRB) of the first Affiliated Hospital of Wenzhou Medical University and conducted in accordance with the Declaration of Helsinki (IRB\#2016054). The need for written informed consent was waived with confirmation of patient data confidentiality by IRB for this retrospective study. All primary tumors were confirmed cytologically or histologically. Total body single-photon emission computed tomography (SPECT) and brain magnetic resonance imaging (MRI) were performed for tumor staging purposes before the start of treatment.

\section{Target definition and treatment planning}

Contrast computed tomography (CT) images were scanned to delineate the gross tumor volume (GTV) which encompasses the primary tumor volume and the positive mediastinal lymph nodes according to the International Commission on Radiation Units and Measurements (ICRU) report 62 criteria. ${ }^{17}$ The clinical target volume (CTV) was generated by including GTV and uninvolved mediastinal and ipsilateral hilar nodes. CTV added a 7-mm margin to generate a planning target volume (PTV). OARs, such as lungs and heart, were automatically contoured using the Pinnacle $^{3}$ treatment planning system (version 9.8; Philips Radiation Oncology Systems, Fitchburg, USA) with necessary manual correction. The inner margin of the entire bony thoracic spinal canal was defined as the spinal cord. The length of the esophagus was contoured from the lower border of the cricoid cartilage to the gastro-esophageal junction.

For VMAT plan optimization, single or two-arc VMAT plans were generated according to target volume and dosimetric considerations at a synergy accelerator (Elekta, Stockholm Sweden) equipped with MLCi2 multileaf collimator (MLC). The prescription dose for these patients was 2 Gy for 2632 fractions at $6 \mathrm{MV}$.A final arc space degree of 4 was employed with a leaf motion of $0.46 \mathrm{~cm} / \mathrm{deg}$. For one arc plans, the start and stop angles were $181^{\circ}$ and $180^{\circ}$ with clockwise $(\mathrm{CW})$ rotation direction. Another counterclockwise arc from $180^{\circ}$ to $181^{\circ}$ was added to make a two-arc plan. Dosimetric constraints on PTV coverage and OAR sparing were setup for plan optimization. The coverage of PTV was set to at least $95 \%$ of the PTV volume covered by the 95\% prescription dose. No point dose of the spinal cord should exceed 45 Gy. The V20 and V30 (the percent volume of lung receiving $20 \mathrm{~Gy}$ and $30 \mathrm{~Gy}$ ) should not exceed $30 \%$ and $20 \%$, respectively.

\section{Assessment of pulmonary toxicities}

Patients were followed up and evaluated weekly with a chest radiograph or CT scan during treatment, 3 to 4 weeks after treatment completion, monthly in the first half year, and then every 3 months to 2 years, and every 6 months thereafter. Patients were required to have an immediate examination or intervention if 
symptoms, such as fever, cough or shortness of breath were observed during follow-up. Acute and late radiation pneumonitis (RP) were diagnosed by at least two radiation oncologists with consensus. RP was scored as greater than or equal to grade III side effects according to the Common Terminology Criteria for Adverse Events (CTCAE) version 3.0.18

\section{Statistical analysis}

The records of enrolled patients were retrospectively reviewed until September 31, 2016. The end points of this study were OS and progression-free survival (PFS). PFS was evaluated based on clinical examination. If metastases were suspected, more images were required. The OS rate was analyzed with the Kaplan-Meier test. The association between dosimetric factors and RP were investigated by univariate and multivariate logistic regression. For variables that showed a trend toward statistical significance in the univariate analysis, forward stepwise logistic regression analysis was applied in the further multivariate analysis. SPSS 17.0 (IBM, Armonk, NY, USA.) was applied for statistical analyses, and $p<0.05$ was set as statistical significance.

\section{Results}

A total of 134 lung cancer patients were enrolled in this study with a mean age of 62.6 years (range, $40-86$ y). The average prescription dose to these patients was 57.07 \pm 6.27 Gy (range, 52-64 Gy). The overall median follow-up time was 18.6 months (range, 2-45 mo). The median follow-up time of the surviving patients was 20 months (range, 7-45 mo). The patient characteristics and lung dosimetric values are listed in Table 1.

The two-year PFS rate for all patients was $18.2 \%$ with a median PFS time of 7.6 months, as shown in Fig. $1 \mathrm{~b}$. The 2-year OS for all patients was $38.4 \%$, with a median survival time of 18.6 months, as shown in Fig. 1a. The median OS for stage 1, 2, 3 and 4 patients were $21.9,15.8,23.1$, and 11.8 months, respectively. There were no significant survival differences among patients of all stages.

Table 1. The characteristics of non-small-cell lung cancer patients enrolled

\begin{tabular}{ll}
\hline Characteristics & $\mathrm{N}=134(\%)$ \\
\hline Gender & $101(75.3)$ \\
Male & $33(24.7)$ \\
Female & $62.6 \pm 10.1$ \\
Age (years) & \\
Histological subtype & $25(18.7)$ \\
Small cell & $54(40.3)$ \\
Squamous carcinoma & $46(34.3)$ \\
Adenocarcinoma & $9(6.7)$ \\
Others & $43(32.1)$ \\
Tumor location & $12(9.0)$ \\
Left upper lobe & $55(41.0)$ \\
Left lower lobe & $24(17.9)$ \\
Right upper lobe & \\
Right lower lobe & \\
Stage & $12(9.0)$ \\
1 & $6(4.5)$ \\
2 & $63(47.0)$ \\
3 & $53(39.5)$ \\
4 & \\
Chemotherapy & $110(82.1)$ \\
Yes & $24(17.9)$ \\
No & $57.07 \pm 6.27$ \\
Average dose Prescription (Gy) & \\
Lung dosimetry & $59.9 \pm 18.2$ \\
V5 (\%) & $44.6 \pm 16.0$ \\
V10 (\%) & $36.9 \pm 13.3$ \\
V13 (\%) & $24.6 \pm 8.5$ \\
V20 (\%) & $16.0 \pm 5.3$ \\
V30 (\%) & $15.08 \pm 3.31$ \\
Mean dose (Gy) & \\
Heart dosimetry & $25.8 \pm 17.4$ \\
V25 (\%) & $20.8 \pm 14.2$ \\
V30 (\%) & $6.8 \pm 6.9$ \\
V50 (\%) & $15.20 \pm 8.61$ \\
Mean dose (Gy) & \\
PTV parameters & $408.2 \pm 325.1$ \\
Volume (cm $)$ & $54.91 \pm 9.57$ \\
Mean dose (Gy) & $60.61 \pm 8.69$ \\
Max dose (Gy) & \\
\hline & \\
& \\
\hline
\end{tabular}

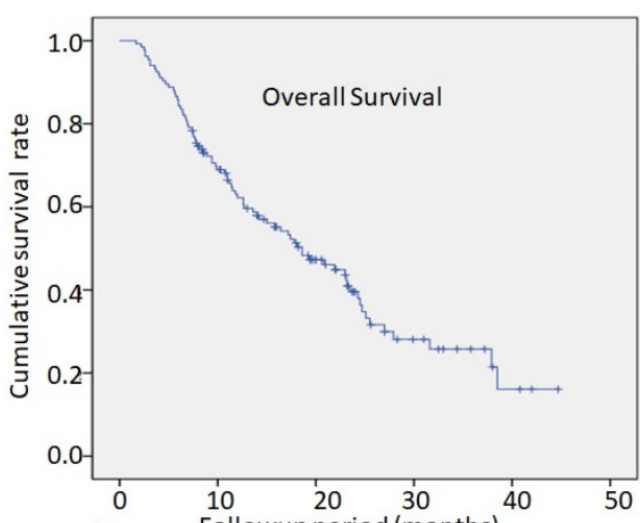

a)

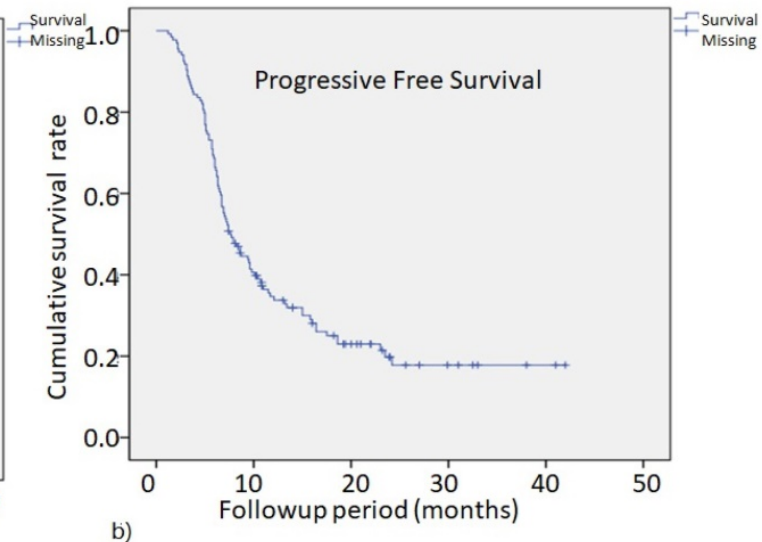

b)

Figure 1. The overall survival (OS) for all patients at 2 years was $38.4 \%$, with a median survival time of 18.6 months (Fig.la). The progression-free survival (PFS) rate in all patients at 2 years was $18.2 \%$, with a median PFS time of 7.6 months (Fig. 1 b). 


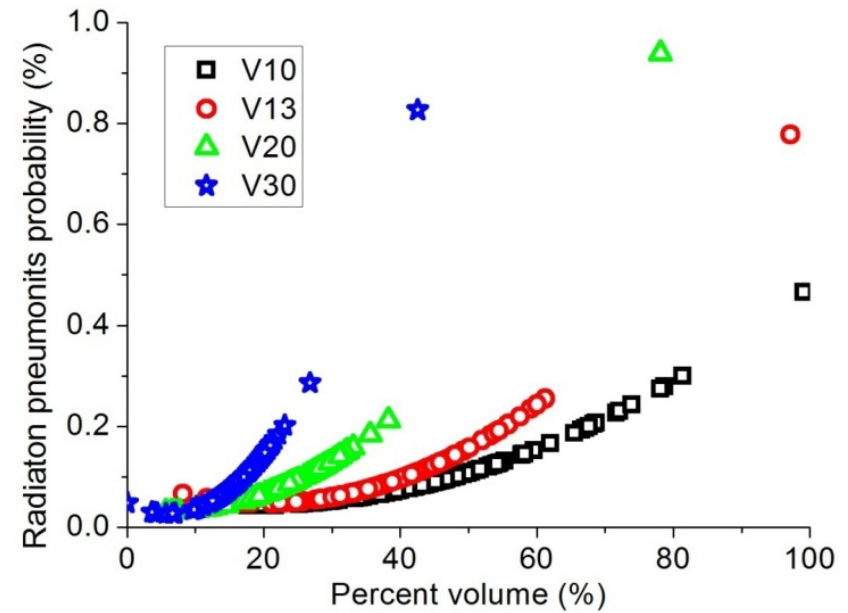

Figure 2. Regression analysis results to limit the RP rate to less than $10 \%$ with the constraints for $\mathrm{V} 10, \mathrm{~V} 13, \mathrm{~V} 20$, and $\mathrm{V} 30$ of $49 \%, 41 \%, 26 \%$, and $17 \%$, respectively.

Dosimetric values of mean lung dose (MLD) and percent lung volume irradiated by a certain dose (V5-30), heart dose, PTV dose and tumor location, as well as univariate analyses on factors related to acute
$\mathrm{RP}$, are shown in Table 2. Fourteen of 134 patients had grade III/higher RP (10.5\%). There were no acute treatment-related deaths. Age, chemotherapy exposure, dose prescription, V10, V13, V20, and V30 were significantly associated with acute RP. Sex, pathological type, stage, V5, and mean lung dose were not associated with acute RP in the univariate analysis. In the multivariate analysis, only V13 $(p=0.02)$ and age $(p=0.02)$ were independently associated with acute RP. To limit the RP rate to less than $10 \%$, the constraint of V10, V13, V20, and V30 were approximately $49 \%, 41 \%, 26 \%$, and $17 \%$, respectively, as shown in Figure 2.

Dosimetric values and univariate analysis results for patients with pulmonary fibrosis are summarized in Table 2. Twelve patients developed grade III/higher pulmonary fibrosis $(9.0 \%)$. Univariate analyses showed that dose prescription and PTV volume were significantly associated with chronic pneumonitis.

Table 2. Univariate analysis for factors associated with acute radiation pnuemonitis and fibrosis

\begin{tabular}{|c|c|c|c|c|c|c|c|c|}
\hline \multirow[t]{3}{*}{ Factors } & \multicolumn{4}{|c|}{ Radiation pneumonitis } & \multicolumn{4}{|l|}{ Fibrosis } \\
\hline & \multirow{2}{*}{$\begin{array}{l}\text { Grade } 0-2(n, \%) \\
N=120\end{array}$} & \multirow{2}{*}{$\begin{array}{l}\text { Grade 3-4(n,\%) } \\
\mathrm{N}=14\end{array}$} & \multirow[t]{2}{*}{$x^{2}$} & \multirow[t]{2}{*}{$P$} & \multirow[t]{2}{*}{ Grade $0-2(n, \%) N=122$} & \multirow{2}{*}{$\begin{array}{l}\text { Grade } 3-4(n, \%) \\
N=12\end{array}$} & \multirow[t]{2}{*}{$x^{2}$} & \multirow[t]{2}{*}{$\mathrm{P}$} \\
\hline & & & & & & & & \\
\hline \multicolumn{9}{|l|}{ Gender } \\
\hline Male & $90(75.0)$ & $11(78.6)$ & 0.086 & 0.77 & $91(74.6)$ & $10(83.3)$ & 0.45 & 0.5 \\
\hline Female & $30(25.0)$ & $3(21.4)$ & & & $31(25.4)$ & $2(16.7)$ & & \\
\hline Age (years) & $61.8 \pm 10.1$ & $69.1 \pm 8.4$ & 2.58 & 0.01 & $62.3 \pm 10.2$ & $66.5 \pm 8.5$ & 1.39 & 0.17 \\
\hline \multicolumn{9}{|l|}{ Histological subtype } \\
\hline Small cell & $21(17.5)$ & $4(28.6)$ & 1.61 & 0.66 & $23(18.9)$ & $2(16.7)$ & 2.34 & 0.51 \\
\hline Squamous carcinoma & $48(40.0)$ & $6(42.9)$ & & & 47 (38.5) & $7(58.3)$ & & \\
\hline Adenocarcinoma & $43(35.8)$ & $3(21.4)$ & & & $44(36.1)$ & $2(16.7)$ & & \\
\hline Others & $8(6.7)$ & $1(7.1)$ & & & $8(6.6)$ & $1(8.3)$ & & \\
\hline \multicolumn{9}{|l|}{ Tumor location } \\
\hline Left upper lobe & $39(32.5)$ & $4(28.6)$ & 1.87 & 0.6 & $40(32.8)$ & $3(25.0)$ & 0.48 & 0.92 \\
\hline Left lower lobe & $12(10.0)$ & $0(0)$ & & & $11(9.0)$ & $1(8.3)$ & & \\
\hline Right upper lobe & $48(40.0)$ & $7(50.0)$ & & & 49 (40.2) & $6(50.0)$ & & \\
\hline Right lower lobe & $21(17.5)$ & $3(21.4)$ & & & $22(18.0)$ & $2(16.7)$ & & \\
\hline \multicolumn{9}{|l|}{ Stage } \\
\hline 1 & $11(9.2)$ & $1(7.1)$ & 1.16 & 0.76 & $11(9.0)$ & $1(8.3)$ & 1.04 & 0.79 \\
\hline 2 & $5(4.2)$ & $1(7.1)$ & & & $6(4.9)$ & $0(0.0)$ & & \\
\hline 3 & $58(48.3)$ & $5(35.7)$ & & & $58(47.5)$ & $5(41.7)$ & & \\
\hline 4 & $46(38.3)$ & $7(50.0)$ & & & 47 (38.5) & $6(50.0)$ & & \\
\hline \multicolumn{9}{|l|}{ Chemotherapy } \\
\hline Yes & $102(85.0)$ & $8(57.1)$ & 6.62 & 0.01 & $100(82.0)$ & $10(83.3)$ & 0.01 & 0.91 \\
\hline No & $18(15.0)$ & $6(42.9)$ & & & $22(18.0)$ & $2(16.7)$ & & \\
\hline Prescription dose (Gy) & $56.81 \pm 6.56$ & $59.36 \pm 1.45$ & 3.58 & 0.001 & $56.85 \pm 6.49$ & $59.35 \pm 2.25$ & 2.85 & 0.007 \\
\hline \multicolumn{9}{|l|}{ Lung dosimetries } \\
\hline V5 (\%) & $58.9 \pm 18.00$ & $68.9 \pm 18.7$ & 1.75 & 0.08 & $59.8 \pm 17.7$ & $60.9 \pm 23.7$ & 0.18 & 0.86 \\
\hline V10 (\%) & $43.5 \pm 15.4$ & $53.9 \pm 18.9$ & 2.05 & 0.04 & $44.4 \pm 16.0$ & $46.1 \pm 17.1$ & 0.31 & 0.76 \\
\hline V13 (\%) & $35.8 \pm 12.2$ & $45.5 \pm 19.2$ & 2.33 & 0.02 & $36.8 \pm 13.4$ & $37.3 \pm 13.1$ & 0.12 & 0.91 \\
\hline V20 (\%) & $23.9 \pm 7.0$ & $31.0 \pm 16.1$ & 2.7 & 0.008 & $24.7 \pm 8.7$ & $23.8 \pm 7.0$ & 0.32 & 0.75 \\
\hline V30 (\%) & $15.6 \pm 4.8$ & $19.6 \pm 7.9$ & 2.45 & 0.02 & $16.1 \pm 5.4$ & $15.6 \pm 4.7$ & 0.28 & 0.78 \\
\hline Mean dose (Gy) & $14.99 \pm 3.38$ & $15.84 \pm 2.68$ & 0.81 & 0.42 & $15.18 \pm 3.36$ & $14.10 \pm 2.83$ & 0.98 & 0.33 \\
\hline \multicolumn{9}{|l|}{ Heart dosimetries } \\
\hline V25 (\%) & $25.9 \pm 17.7$ & $24.7 \pm 15.2$ & 0.055 & 0.82 & $25.6 \pm 17.8$ & $27.2 \pm 15.5$ & 0.12 & 0.73 \\
\hline V30 (\%) & $20.8 \pm 14.3$ & $21.2 \pm 14.1$ & 0.014 & 0.91 & $20.57 \pm 14.4$ & $22.3 \pm 13.4$ & 0.21 & 0.65 \\
\hline V50 (\%) & $6.8 \pm 6.8$ & $7.5 \pm 7.5$ & 0.15 & 0.70 & $6.7 \pm 6.8$ & $7.8 \pm 7.3$ & 0.38 & 0.54 \\
\hline Mean dose (Gy) & $15.16 \pm 8.64$ & $15.56 \pm 8.63$ & 0.025 & 0.87 & $14.99 \pm 8.67$ & $16.52 \pm 8.33$ & 0.46 & 0.5 \\
\hline \multicolumn{9}{|l|}{ PTV parameters } \\
\hline Volume (cm3) & $463.4 \pm 321.9$ & $615.7 \pm 330.2$ & 2.97 & 0.09 & $458.8 \pm 287.0$ & $620.4 \pm 458.5$ & 3.94 & 0.049 \\
\hline Mean dose (Gy) & $55.32 \pm 8.30$ & $51.44 \pm 16.99$ & 1.93 & 0.17 & $54.78 \pm 9.80$ & $55.71 \pm 8.21$ & 0.14 & 0.71 \\
\hline Max dose (Gy) & $60.34 \pm 8.83$ & $62.85 \pm 8.69$ & 0.98 & 0.32 & $60.38 \pm 8.76$ & $62.05 \pm 8.39$ & 0.54 & 0.46 \\
\hline
\end{tabular}




\section{Discussion}

Although dosimetric studies had reported that VMAT can be delivered safely with acceptable acute and late toxicities for lung cancer, this is one of the few clinical evidences presented with the clinical outcome, as well as the dosimetric effects, of NSCLC patients who underwent VMAT.

Planning studies had evaluated VMAT techniques in lung cancer and reported that IMRT and VMAT resulted in a better conformity of dose and allowed higher dose to the target volume, thus improving regional tumor control. ${ }^{19}$ Planning studies had demonstrated that there is a dosimetric advantage of using VMAT compared with 3DCRT, and MU and delivery efficiency advantage compared with IMRT for lung cancer. However, there are no published randomized trials comparing the clinical outcome and toxicity between 3DCRT and IMRT/VMAT or IMRT versus VMAT in NSCLC, and such studies will not be forthcoming. ${ }^{20}$ The overall 2-year PFS and OS reported in this study were 18.2\% and $38.4 \%$, respectively. In the literature, Memorial Sloan Kettering study reported a 2-year OS of $58 \%$ and a median survival time of 25 months for 55 patients with stages Ib-IIIb (62\% stage III) NSCLC. ${ }^{21}$ Yom et al reported a local control of $55.3 \%$ at 12 months with an estimated OS of $57 \%$ for 68 NSCLC patients (85\% stage III) who underwent IMRT. ${ }^{22}$ Liao et al reported a 2 -year DFS and OS of $38 \%$ and $46 \%$, respectively, for 91 patients with locally advanced NSCLC treated by IMRT for a median 16.5 months of follow-up. ${ }^{23}$ These variations in PFS and OS from various studies may attributed to different factors such as adjuvant chemotherapy, plan optimization technique, experience of the treatment planner, treatment volume margins, dose prescription, location of the tumor, and dose calculation algorithms.

Studies had reported that the OS of NSCLC patients who underwent IMRT is not inferior to that of those treated by 3D-CRT, but the risk of RP was significantly reduced and the risk of radiation esophagitis increased by IMRT compared with 3D-CRT. ${ }^{24}$ IMRT had a significantly $(p=0.002)$ lower level of grade $\geq 3$ radiation pneumonitis than $3 \mathrm{D}-\mathrm{CRT}$ at 12 months (according to RTOG toxicity scoring), with $8 \%$ [95\% confidence interval (CI): $4 \%-19 \%$ ] and $32 \%(95 \%$ CI: $26 \%-40 \%)$ for IMRT and 3D-CRT, respectively. ${ }^{25}$ On the other hand, great concern had been focused on the fatal RP toxicity associated with IMRT. ${ }^{26}$ However, the RP rate of lung VMAT treatment had been rarely reported. In this study, a RP rate of $10.4 \%$ was observed in patients who underwent VMAT.

The effect of lung dose-volume parameters on the development of RP has been long investigated.
Different constraints for different lung dose-volume histogram (DVH) parameters, such as V5, V10, V13, V20, V30, V40, and LMD, have been suggested to reduce the volume of normal lung irradiated and decrease the RP rate. ${ }^{27,28}$ In this study, in addition to age, chemotherapy exposure, and dose prescription, lung DVH parameters of V10, V13, V20, and V30 were observed to associated with RP. However, the high association of V5 with fatal RP reported in the IMRT treatment of pleural mesothelioma was not observed in this study. 27 Similarly, in a previous study analyzing the dosimetric effects on 94 NSCLC patients treated by concurrent chemotherapy and IMRT, V5 was reported to be associated with severe acute RP in univariate analysis, but not in multivariate analysis. ${ }^{29}$ To limit the RP rate to less than $10 \%$, the constraint of V10, V13, V20 and V30 were approximately $49 \%, 41 \%$, $26 \%$, and $17 \%$, respectively, for patients who underwent VMAT.

This is a retrospective study from our institution only, we may not be able to fully evaluate the impact of various treatment-related outcomes in this study, such as chemotherapy. Chemotherapy had been reported to play an important role and increase the risk of RP in NSCLC treatment. ${ }^{30}$ More patients with longer follow-up duration are needed to further evaluate the efficacy and toxicities of VMAT in the treatment of NSCLC.

\section{Conclusions}

The treatment effects and toxicities reviewed in this article demonstrated that VMAT can be delivered safely with acceptable acute and late toxicities. Lung dosimetric metrics were valuable in predicting acute RP. A constraint of $40 \%$ of lung V13 in the VMAT treatment of NSCLC was helpful to limit the RP rate to less than $10 \%$.

\section{Acknowledgements}

The study was partially supported by grants from the National Natural Science Foundation of China (11675122, 2017), the Natural Science Foundation of Zhejiang Province (LY17H160051, 2017) and Wenzhou Municipal Science and Technology Bureau (H20100068, 2010).

\section{Competing Interests}

The authors have declared that no competing interest exists.

\section{References}

1. Furuse K, Fukuoka M, Kawahara M, et al. Phase III study of concurrent versus sequential thoracic radiotherapy in combination with mitomycin, vindesine, and cisplatin in unresectable stage III nonsmall-cell lung cancer. J Clin Oncol. 1999;17:2692-2699. 
2. Liu HH, Wang X, Dong L, et al. Feasibility of sparing lung and other thoracic structures with intensity-modulated radiotherapy for non-small-cell lung cancer. Int J Radiat Oncol Biol Phys. 2004;58:1268-1279.

3. Murshed H, Liu HH, Liao Z, et al. Dose and volume reduction for normal lung using intensity-modulated radiotherapy for advanced-stage nonsmall-cell lung cancer. Int J Radiat Oncol Biol Phys. 2004;58:1258-1267.

4. Simeonova A, Abo-Madyan Y, El-Haddad M, et al. Comparison of anisotropic aperture based intensity modulated radiotherapy with 3D-conformal radiotherapy for the treatment of large lung tumors. Radiother Oncol. 2012;102:268-273.

5. Chan C, Lang S, Rowbottom C, Guckenberger M, Faivre-Finn C; IASLC Advanced Radiation Technology Committee. Intensity-Modulated Radiotherapy for Lung Cancer: Developments. J Thorac Oncol. 2014;9(11):1598-608.

6. Sura S, Gupta V, Yorke E, Jackson A, Amols H, Rosenzweig KE. Intensity modulated radiation therapy (IMRT) for inoperable non-small cell lung cancer: the Memorial Sloan-Kettering Cancer Center (MSKCC) experience. Radiother Oncol. 2008, 87:17-23.

7. Liao ZX, Komaki RR, Thames HD Jr, et al: Influence of technologic advances on outcomes in patients with unresectable, locally advanced non-small-cell lung cancer receiving concomitant chemoradiotherapy. Int J Radiat Oncol Biol Phys. 2010, 76:775-781.

8. Guerrero Urbano MT, Nutting CM. Clinical use of intensity- modulated radiotherapy: Part II. Br J Radiol. 2004;77:177-82. 9.

9. Podgarsak EB. Special procedures and techniques in radiotherapy. Radiation Oncology Physics-A Handbook for Teachers and Student, First Edition. Vienna, Austria: IAEA Publications; 2008; 538-47.

10. Hall EJ, Wuu CS. Radiation-induced second cancers: The impact of 3D-CRT and IMRT. Int J Radiat Oncol Biol Phys. 2003;56:83-8.

11. Ruben JD, Davis S, Evans C, et al. The effect of intensity-modulated radiotherapy on radiation-induced second malignancies. Int J Radiat Oncol Biol Phys. 2008;70:1530-6.

12. Ott o K. Volumetric modulated arc therapy: IMRT in a single gantry arc. Med Phys. 2008;35:310-7.

13. Yu CX, Li XA, Ma L, et al. Clinical implementation of intensity-modulated arc therapy. Int J Radiat Oncol Biol Phys. 2002;53:453-63.

14. Palma DA, Verbakel WF, Ott o K, Senan S. New developments in arc radiation therapy: A review. Cancer Treat Rev. 2010;36:393-9

15. Hoogeman MS, Nuytt ens JJ, Levendag PC, Heijmen BJ. Time dependence of intrafraction patient motion assessed by repeat stereoscopic imaging. Int J Radiat Oncol Biol Phys. 2008;70:609-18

16. Ong CL, Verbakel WF, Cuijpers JP, Slotman BJ, Lagerwaard FJ, Senan S. Stereotactic radiotherapy for peripheral lung tumors: A comparison of volumetric modulated arc therapy with 3 other delivery techniques. Radiother Oncol. 2010; 97:437-42.

17. International Commission on Radiation Units and Measurements. ICRU Reports 62 (Supplement to ICRU report 50). Prescribing, recording, and reporting photon beam therapy. Bethesda, MD: ICRU, 1999.

18. Trotti A, Colevas AD, Setser A, et al. CTCAE v3.0: Development of a comprehensive grading system for the adverse effects of cancer treatment. Semin Radiat Oncol. 2003;13:176-181.

19. Bree Id, van Hinsberg MG, van Veelen LR. High-dose radiotherapy in inoperable non-small cell lung cancer: Comparison of volumetric modulated arc therapy, dynamic IMRT and 3D conformal radiotherapy. Med Dosim. 2012;37:353-7.

20. Bezjak A, Rumble RB, Rodrigues G, Hope A, Warde P. Members of the IMRT Indications Expert Panel. Intensity-modulated radiotherapy in the treatment of lung cancer. Clin Oncol. 2012;24:508-520

21. Sura S, Gupta V, Yorke E, Jackson A, Amols H, Rosenzweig KE. Intensity-modulated radiation therapy (IMRT) for inoperable non-small cell lung cancer: the Memorial Sloan-Kettering Cancer Center (MSKCC) experience. Radiother Oncol. 2008;87:17-23

22. Yom SS, Liao $\mathrm{Z}$, Liu $\mathrm{HH}$, et al. Initial evaluation of treatment-related pneumonitis in advanced-stage non-small-cell lung cancer patients treated with concurrent chemotherapy and intensity-modulated radiotherapy. Int J Radiat Oncol Biol Phys. 2007:68:94-102.

23. Liao ZX, Komaki RR, Thames HD Jr, et al. Influence of technologic advances on outcomes in patients with unresectable, locally advanced non-small-cell lung cancer receiving concomitant chemoradiotherapy. Int J Radiat Oncol Biol Phys. 2010;76:775-781

24. $\mathrm{Hu} \mathrm{X}, \mathrm{He} \mathrm{W}$, Wen $\mathrm{S}$,et al. Is IMRT superior or inferior to 3DCRT in radiotherapy for NASCLC? A meta-analysis. PloS One. 2016; 11(4):e0151988.

25. [Internet] RTOG Acute Radiation Morbidity Scoring Criteria. http://www.rtog.org/Research Associates / Adverse Event Reporting / Acute Radiation Morbidity Scoring Criteria.aspx.

26. Yom SS, Liao $\mathrm{Z}$, Liu $\mathrm{HH}$, et al. Initial evaluation of treatment-related pneumonitis in advanced-stage non-small-cell lung cancer patients treated with concurrent chemotherapy and intensity-modulated radiotherapy. Int J Radiat Oncol Biol Phys. 2007, 68:94-102.

27. Allen AM, Czerminska M, Jänne PA, et al. Fatal pneumonitis associated with intensity-modulated radiation therapy for mesothelioma. Int J Radiat Oncol Biol Phys. 2006;65:640-645.

28. Rodrigues G, Lock M, D'Souza D, Yu E, Van Dyk J. Prediction of radiation pneumonitis by dose-volume histogram parameters in lung cancer-a systematic review. Radiother Oncol. 2004;71(2):127-38.
29. Shi A, Zhu G, Wu H, Yu R, Li F, Xu B. Analysis of clinical and dosimetric factors associated with severe acute radiation pneumonitis in patients with locally advanced non-small cell lung cancer treated with concurrent chemotherapy and intensity-modulated radiotherapy. Radiat Oncol. 2010;5:35.

30. Zhang XJ, Sun JG, Sun J, et al. Prediction of radiation pneumonitis in lung cancer patients: a systematic review. J Cancer Res Clin Oncol. 2012;138(12):2103-16. 